\title{
AVALIAÇÃO COMPARATIVA ENTRE ACEPROMAZINA, DETOMIDINA E ROMIFIDINA EM EQÜINOS
}

\author{
COMPARATIVE EVALUATION OF ACEPROMAZINE, \\ DETOMIDINE AND ROMIFIDINE IN HORSES
}

\author{
Denise Tabacchi Fantoni $^{1}$ Fábio Futema ${ }^{2}$ Sílvia Renata Gaido Cortopassi ${ }^{3}$ \\ Luís Cláudio Lopes Correia da Silva ${ }^{2}$ Manoel Verenguer ${ }^{4}$ \\ Regina Mirandola ${ }^{5}$ Márcio Augusto Ferreira ${ }^{2}$
}

RESUMO

Os efeitos da acepromazina, detomidina e romifidina em eqüinos foram comparados, objetivando-se determinar qual o agente mais seguro e efetivo para a referida espécie animal. Foram utilizados 15 eqüinos hígidos, de ambos os sexos, idades e raças variadas e encaminhados ao setor de Cirurgia do Hospital Veterinário da FMVZ - USP. Os animais foram distribuídos em três grupos: grupo I, recebeu $0,1 \mathrm{mg} / \mathrm{kg}$ de acepromazina; grupo II recebeu $20 \mathrm{mcg} / \mathrm{kg}$ de detomidina e grupo III, o qual recebeu $80 \mathrm{mcg} / \mathrm{kg}$ de romifidina, sendo todos os agentes administrados através da via intravenosa. Previamente à administração dos fármacos e aos 15, 30, 60 e 90 minutos, após sua aplicação, foram avaliadas a freqüência e ritmo cardíacos, freqüência respiratória, pressão arterial sistólica e diastólica, assim como foram coletadas amostras de sangue arterial para verificação do $\mathrm{pH}$, pressão parcial de oxigênio e pressão parcial de dióxido de carbono. Os resultados foram submetidos à análise estatística através do teste de análise de variância (ANOVA), seguido do teste de Tukey. Não foi observada nenhuma alteração da freqüência respiratória nos grupos estudados. Verificou-se diminuição significativa dos valores da FC nos animais tratados com os agentes $\alpha-2$ agonistas quando comparados com os valores obtidos no grupo I. Após a administração da acepromazina, observou-se diminuição significativa das pressões sistólica $e$ diastólica em relação aos valores controle. Os animais do grupo I apresentaram valores pressóricos estatisticamente inferiores em relação aos valores obtidos nos grupos II e III. Os animais do grupo II demonstraram incremento da pressão arterial sistólica após 15 minutos da aplicação do agente, com subseqüente redução destes valores. O mesmo ocorreu no grupo III, porém, sem valor estatístico significativo. Com estes resultados, pode-se concluir que a romifidina e a detomidina promoveram alterações paramétricas de menor intensidade e duração quando comparadas à acepromazina.

Palavras-chave: acepromazina, romifidina, detomidina, eqüinos.

SUMMARY

The effects of acepromazine, detomidine and romifidine were compared in horses in the present study, aiming to determine which agent is safer and more effective for the referred species of animal. Fifteen healthy horses of the same sex, age and breed varying, were sent to the Surgical Division of the Veterinarian Hospital of FMVZ-USP. The animals were distributed in three groups; group I - GI - received $0.1 \mathrm{mg} / \mathrm{kg}$ of acepromazine; group II - GII - received $20 \mu \mathrm{g} / \mathrm{kg}$ of detomidine; group III - GIII received $80 \mu \mathrm{g} / \mathrm{kg}$ of romifidine, all agents delivered intravenously. Prior to administration of the agents and at 15, 30, 60 and 90 minutes after their application, the following parameters were evaluated: heart rate, respiratory rate, systolic and diastolic arterial pressure. Arterial blood samples were collected to verify $\mathrm{pH}$, partial oxygen pressure and partial carbon dioxide pressure. The results were submitted to statistical analysis using the analysis of variance (ANOVA) followed by the Tukey test. No significant alteration was observed in respiratory rate of the studied groups. The heart rate significantly decreased in the animals treated with the alpha-2 agonist agents when compared to the values obtained in the GI. Following administration of acepromazine a significant decrease $n$ systolic and diastolic blood pressures was observed in relation to control values. The animals of GI presented pressure values that were statistically inferior in relation to the values obtained for groups II and III. The animals in GII showed an increase in systolic

\footnotetext{
${ }^{1}$ Médico Veterinário, Professor Doutor, Faculdade de Medicina Veterinária e Zootecnia (FMVZ), Universidade de São Paulo (USP). Rua Guarará, 538 apto 151, 01425-000, São Paulo, SP. Autor para correspondência.

${ }^{2}$ Médicos Veterinários, Pós-graduandos do Departamento de Cirurgia, FMVZ, USP.

${ }^{3}$ Médico Veterinário, Professor Doutor, Faculdade de Medicina Veterinária, UNIP e FEOB.

${ }^{4}$ Médico Veterinário Autônomo.

${ }^{5}$ Farmacêutica, Técnica de Nível Superior, FMVZ, USP. 
arterial pressure after 15 minutes of agent administration, with subsequent reduction in this data. The same occurred in GIII however without statistically significant difference. With this results it can be concluded that romifidine and detomidine promoted alterations of less intensity and duration in the studied parameters when compared to acepromazine.

Key words: acepromazine, romifidine, detomidine, horses

\section{INTRODUÇÃO}

O tamanho e temperamento do eqüino fazem com que os procedimentos anestésicos sejam potencialmente perigosos para o animal e para o médico veterinário. A anestesia em eqüinos deve ser caracterizada por indução rápida e suave, requerer pequenas quantidades do agente indutor, produzir adequado relaxamento muscular, proporcionar analgesia, não alterar significativamente os parâmetros cardiopulmonares e, por fim, proporcionar uma recuperação livre de excitação.

A acepromazina é o derivado fenotiazínico mais comumente utilizado como medicação préanestésica na Medicina Veterinária (LUMB \& JONES, 1984). Promove ptose palpebral, ligeira protusão da membrana nictitante, prolapso peniano e abaixamento da cabeça (BOOTH \& MCDONALD, 1992). Seu principal efeito hemodinâmico é a hipotensão arterial. Doses clinicamente recomendadas reduzem a pressão arterial em 15 a $20 \mathrm{mmHg}$ do basal. Esta redução da pressão arterial é dosedependente, podendo levar à taquicardia reflexa e aumento da concentração de catecolaminas circulantes. A hipotensão ocorre devido ao bloqueio alfaadrenérgico periférico e à depressão hipotalâmica (MUIR \& HUBBELL,1991).

A diminuição da pressão venosa central está relacionada diretamente com o aumento da frequiência cardíaca, combinada com a redução da resistência vascular sistêmica, resultando no aumento inicial do débito cardíaco (MUIR \& MASON,1993). Outros efeitos encontrados com a administração da acepromazina são depressão miocárdica, diminuição da temperatura corporal, aumento da perfusão cutânea e visceral, ação antiarrítimica, diminuição da concentração da hemoglobina, vasodilatação esplênica e mínima depressão respiratória (GEISER, 1990).

Os fármacos pertencentes ao grupo dos agentes $\alpha-2$ agonistas são utilizados atualmente com maior freqüência no eqüino como sedativos e/ou analgésicos (MUIR \& HUBBELL,1991). Estes agentes produzem sedação e relaxamento muscular bem mais pronunciados do que outros agentes sedativos utilizados na medicação pré-anestésica no eqüino (MUIR \& HUBBELL, 1991). Os efeitos de sua ação no sistema nervoso central incluem sedação, hipnose, relaxamento muscular, ataxia e analgesia (GEISER, 1990). Os efeitos cardiopulmonares incluem diminuição da frequiência cardíaca, bloqueio átrio-ventricular, redução do débito cardíaco, aumento transitório da pressão arterial, seguida de hipotensão. Estes efeitos são toleráveis em eqüinos com função cardiovascular normal (WAGNER et al.,1991).

A detomidina é um dos agentes $\alpha$ - 2 agonistas mais comumente utilizado na sedação e medicação pré-anestésica em eqüinos(CHAMBERS $\boldsymbol{e t}$ al., 1993), podendo ser recomendada nas intervenções cirúrgicas em eqüinos na posição quadrupedal (FEITOSA et al., 1990). Outros efeitos observados com a utilização dos agentes $\alpha$-2 agonistas são prolapso peniano, ataxia, ptose labial, abaixamento da cabeça, anorexia, glicosúria, diminuição da motilidade intestinal, aumento da tonicidade uterina, diminuição do hematócrito e relativo aumento da glicose sangüínea, estes dois últimos, respectivamente, por vasodilatação esplênica e por inibição da secreção de insulina mediada pela estimulação dos $\alpha$-2 adrenoceptores (MUIR \& HUBBELL, 1991).

O escopo do presente estudo foi a comparação entre a romifidina, detomidina e acepromazina, através da avaliação de parâmetros cardiovasculares e ventilatórios.

\section{MATERIAL E MÉTODOS}

Foram utilizados 15 equiinos de ambos os sexos, idades e raças variadas, pertencentes à categoria de risco 1 e 2, e provenientes do setor de Cirurgia do Hospital Veterinário da FMVZ - USP. Os animais foram submetidos a jejum alimentar de 12 horas e hídrico de 4 horas.

Os animais foram distribuídos em três grupos: os animais do grupo I foram tratados com $0,1 \mathrm{mg} / \mathrm{kg}$ de acepromazina ${ }^{\mathrm{a}}$, os animais do grupo II foram tratados com $20 \mathrm{mcg} / \mathrm{kg}$ de detomidina ${ }^{\mathrm{b}}$ e os animais do grupo III foram tratados com $80 \mathrm{mcg} / \mathrm{kg}$ de romifidina ${ }^{c}$, sendo todos os agentes administrados através da via intravenosa.

Avaliou-se freqüência (FC) e ritmo cardíacos através da unidade de tempo minuto, com auxílio de eletrocardiógrafo ${ }^{\mathrm{d}}$ na velocidade de $25 \mathrm{~mm} / \mathrm{sec}$, tendo resultado da divisão do número absoluto 1500 , pelo intervalo R-R em milissegundos, tendo sido realizadas as derivações do plano frontal (bipolares I, II, III e unipolares - avr,avl, avf) em todos os momentos onde se fez o uso do eletrocardiógrafo (ECG). A frequiência respiratória (FR) foi constatada pelo método de auscultação sobre os campos pulmonares; a mensuração das pressões arteriais sistólica 
(PAS) e diastólica (PAD) foram realizadas através do método não invasivo, com a utilização de um estetoscópio ultrassônico ${ }^{\mathrm{e}}$ para a ausculta dos sons de Korotkoff, posicionando o manguito na base da cauda do animal, tendo-se obtido desta maneira a pressão da artéria coccígea. As amostras de sangue, para obtenção dos valores de $\mathrm{pH}$, pressão parcial de oxigênio no sangue arterial $\left(\mathrm{PaO}_{2}\right)$ e a pressão parcial de dióxido de carbono no sangue arterial $\left(\mathrm{PaCO}_{2}\right)$, foram obtidas através da coleta de sangue arterial, por punção da artéria facial do eqüino. A análise das amostras foi realizada imediatamente após a coleta em analisador de $\mathrm{pH}$ e gases sangüíneos ${ }^{\mathrm{f}}$.

Os tempos de registro de FC e ritmos cardíacos, FR, PAS, PAD, pH e gases sanguiíneos foram realizados antes da administração dos fármacos e aos 15, 30, 60 e 90 minutos após sua aplicação. O método estatístico para comparação entre os diferentes momentos dos grupos e a análise comparativa dos diferentes tempos de observação de um mesmo grupo foram realizados com auxílio da análise de variância (ANOVA), seguida do teste de Tukey, sendo o grau de significância estabelecido de 5\% $(\mathrm{p}<0,05)$.

\section{RESULTADOS}

Os animais que receberam detomidina apresentaram diminuição significativa da FC em todos os momentos de avaliação, que seguiram à administração deste agente em relação aos valorescontrole, sendo $\mathrm{p}$ respectivamente $\mathrm{p}<0,001, \mathrm{p}<0,05$, $\mathrm{p}<0,05$ e $\mathrm{p}<0,05$. No grupo II, os valores de FC aos 15 e 30 minutos foram inferiores aos valores basais, sendo a diferença estatisticamente significativa (respectivamente $\mathrm{p}<0,001$ e $\mathrm{p}<0,05)$. Na análise estatística entre os três grupos, os grupos II e III apresentaram valores inferiores com diferença estatisticamente significativa em relação ao grupo I, aos 15 e 30 minutos de observação $(\mathrm{p}<0,05)$. No entanto, verificou-se recuperação dos valores basais ao término do período de avaliação (Tabela 1).

Os animais que receberam acepromazina não apresentaram qualquer alteração do ritmo cardíaco no decorrer do experimento, o mesmo não ocorrendo com os grupos da romifidina e detomidina nos quais foram observados graus variados de bloqueio da condução. O bloqueio átrio-ventricular de segundo grau Mobitz, tipo I, foi a principal arritmia encontrada. Na comparação entre os grupos dos agentes $\alpha-2$ agonistas não foi observada diferença estatisticamente significativa em relação à incidência do bloqueio.

Não houve diferença estatisticamente significativa da FR, tanto na comparação dos diferen- tes tempos de observação de um mesmo grupo, como também na análise entre os tempos dos três grupos experimentais. Porém, foi evidenciada a diminuição deste parâmetro em todos os grupos após a aplicação dos agentes (Tabela 1).

Nos animais tratados com acepromazina, observou-se diminuição da PAS em todos os tempos estudados, após a administração do agente, sendo a diferença estatisticamente significativa somente nos tempos 15, 30 e 60 minutos (respectivamente $\mathrm{p}<0,001, \mathrm{p}<0,05$ e $\mathrm{p}<0,05)$. Quanto à análise comparativa entre os grupos, a acepromazina demonstrou valores significativamente inferiores quando comparados ao grupo da romifidina nos tempos 15, 30 e 60 minutos $(\mathrm{p}<0,05)$ e quando comparados ao grupo II, os animais do grupo I apresentaram valores significativamente inferiores aos 30 minutos $(p<0,05)$. Nos animais tratados com a detomidina, foi observada diminuição significativa dos valores da PAS nos tempos 60, 90 minutos ( $\mathrm{p}<0,05$ ), após a administração do agente em relação aos valores controle. Após 15 minutos da administração deste agente, observouse incremento da PAS, porém, a diferença não foi estatisticamente significativa. Os animais que receberam romifidina não apresentaram alterações significativas dos valores da PAS na análise, comparativamente aos momentos do mesmo grupo. No entanto, 15 minutos após sua administração, observouse elevação dos valores da PAS com redução nos tempos subseqüentes (Tabela 1 e Figura 1).

Em relação a PAD, os animais do grupo I não apresentaram diferenças significativas entre os diferentes momentos, porém, após a administração do agente, os valores de PAD apresentaram queda progressiva em todos os tempos estudados no mesmo grupo. Na análise comparativa entre os grupos, o grupo I apresentou valores inferiores em relação ao grupo III, em todos os tempos estudados $(\mathrm{p}<0,05)$. $\mathrm{Na}$ avaliação comparativa entre os grupos I e II não foi observada diferença estatisticamente significativa nos tempos estudados, com exceção dos valores obtidos aos 30 minutos ( $<<0,05)$, quando verificouse que os animais tratados com acepromazina mostraram valores de PAD inferiores ao da detomidina (Tabela 1).

Os animais do grupo II apresentaram queda progressiva da $\mathrm{PAD}$, sendo significativa somente após 90 minutos $(\mathrm{p}<0,01)$ da administração do agente, em relação aos valores controle. No entanto, estes valores não foram biologicamente expressivos (Tabela 1).

Não se observou qualquer alteração estatisticamente significativa entre os diferentes tempos de avaliação de cada grupo ou entre os três grupos experimentais dos valores de $\mathrm{pH}$ e $\mathrm{PaCO}_{2}$. A de- 
Tabela 1 - Valores médios e desvios-padrão da pressão arterial sistólica $(\mathrm{mmHg})$, pressão arterial diastólica $(\mathrm{mmHg})$, freqüência cardíaca (batimentos por minuto) e freqúência respiratória (movimentos por minuto) dos animais tratados com Acepromazina (Grupo I), Detomidina (Grupo II) e Romifidina (Grupo III).

\begin{tabular}{|c|c|c|c|c|c|c|c|}
\hline \multirow[b]{2}{*}{ Parâmetro } & \multirow[b]{2}{*}{$\begin{array}{c}\text { Momentos } \\
\text { (minutos) }\end{array}$} & \multicolumn{2}{|c|}{ ROMIFIDINA } & \multicolumn{2}{|c|}{ ACEPROMAZINA } & \multicolumn{2}{|c|}{ DETOMIDINA } \\
\hline & & Média & DP & Média & DP & Média & DP \\
\hline \multirow[t]{5}{*}{ PAS } & 0 & 134,00 & 10,49 & 132,40 & 17,44 & 123,80 & 17,25 \\
\hline & 15 & 147,10 & 10,17 & $109,00^{\mathrm{b}}$ & 30,45 & 127,00 & 34,09 \\
\hline & 30 & 130,10 & 21,09 & $90,80^{\mathrm{a} / \mathrm{b}}$ & 1,79 & 116,30 & 19,98 \\
\hline & 60 & 122,50 & 23,05 & $90,80^{\mathrm{b}}$ & 23,07 & 91,50 & 9,44 \\
\hline & 90 & 127,40 & 23,45 & 100,75 & 8,65 & 95,50 & 19,70 \\
\hline \multirow[t]{5}{*}{ PAD } & 0 & 93,90 & 13,75 & 79,80 & 9,42 & 91,20 & 13,50 \\
\hline & 15 & 111,80 & 14,42 & $65,20^{\mathrm{b}}$ & 38,00 & 90,00 & 25,76 \\
\hline & 30 & 95,00 & 22,15 & $61,40^{\mathrm{a} / \mathrm{b}}$ & 14,12 & 88,11 & 16,03 \\
\hline & 60 & 87,70 & 26,04 & $56,40^{\mathrm{b}}$ & 27,54 & 64,00 & 12,36 \\
\hline & 90 & 98,90 & 27,39 & $70,75^{\mathrm{b}}$ & 3,40 & 71,33 & 15,87 \\
\hline \multirow[t]{5}{*}{$\mathrm{FC}$} & 0 & 43,50 & 9,58 & 56,60 & 19,62 & 44,80 & 5,12 \\
\hline & 15 & $26,70^{\mathrm{b}}$ & 3,37 & 58,60 & 17,28 & 28,90 & 4,43 \\
\hline & 30 & $32,40^{\mathrm{b}}$ & 5,19 & 48,20 & 8,41 & 35,40 & 7,82 \\
\hline & 60 & 33,70 & 5,96 & 38,20 & 5,93 & 37,10 & 8,18 \\
\hline & 90 & 34,20 & 6,88 & 42,00 & 10,58 & 37,40 & 4,19 \\
\hline \multirow{5}{*}{ FR } & 0 & 43,50 & 9,58 & 56,60 & 19,62 & 44,80 & 5,12 \\
\hline & 15 & 26,70 & 3,37 & 68,60 & 17,28 & 28,90 & 4,43 \\
\hline & 30 & 32,40 & 5,19 & 48,20 & 8,41 & 35,40 & 7,82 \\
\hline & 60 & 33,70 & 5,96 & 38,20 & 5,93 & 37,10 & 8,18 \\
\hline & 90 & 34,20 & 6,88 & 42,00 & 10,58 & 37,40 & 4,90 \\
\hline
\end{tabular}

PAS - pressão arterial sistólica; PAD- pressão arterial diastólica; FC- freqüência cardíaca; FRfrequiência respiratória; DP- desvio padrão.

a- análise comparativa entre o grupo da acepromazina e o grupo da detomidina $(\mathrm{p}<0,05)$

b- análise comparativa entre o grupo da acepromazina e o grupo da romifidina $(p<0,05)$

$\mathrm{c}$ - análise comparativa entre o grupo da romifidina e o grupo da detomidina $(\mathrm{p}<0,05)$

aos 15 minutos, em relação à acepromazina (Tabela 2).

\section{DISCUSSÃO}

Diversos fármacos podem ser utilizados na medicação pré-anestésica em eqüinos, porém, a maioria dos agentes pré-anestésicos existentes proporcionam efeitos indesejáveis. Assim sendo, neste experimento, foi realizado um estudo comparativo entre a acepromazina, detomidina e a romifidina com o objetivo de se determinar qual o fármaco mais seguro e efetivo nesta espécie. Verificou-se que a freqüência cardíaca, após 15 e 30 minutos da administração dos agentes romifidina e detomidina, diminuíu significativamente em relação aos valores controle, sendo esta diminuição, clinicamente, importante. HAMM \& JÖCHLE (1991) observaram o mesmo comportamento da frequiência cardíaca, porém, a bradicardia foi mais pronunciada com a detomidina do que com a romifidina, não acontecendo o mesmo no estudo

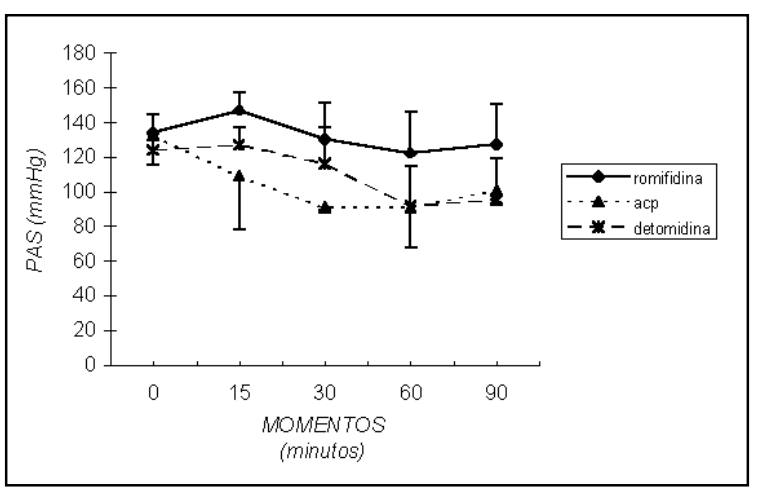

Figura 1 - Representação gráfica da pressão arterial sistólica $(\mathrm{mmHg})$ de eqüinos tratados com acepromazina, romifidina e detomidina no decorrer do tempo (médias e desvios-padrão).

tomidina apresentou os menores valores de $\mathrm{PaO}_{2}$. Em relação aos animais tratados com a romifidina, verificou-se diferença significativa aos 15 e 30 minutos da administração dos dois agentes; e, apenas proposto. WAGNER et al. (1991), ao compararem a xilazina e a detomidina, observaram diminuição da freqüência cardíaca dose-dependente, sendo os efeitos da detomidina mais proeminentes e duradouros. MUIR \& MASON. (1993), ao avaliarem os efeitos da acepromazina na freqüência cardíaca, obtiveram aumento significativo, o mesmo não observado neste experimento.

LUNA et al. (1996), em um estudo comparativo entre detomidina e romifidina em eqüinos, observaram diminuição da freqüência respiratória após a administração de ambos os fármacos em relação aos valores iniciais e ainda, quando esta diminuição foi confrontada entre ambos, observaram-se menores valores de freqüência respiratória nos animais que receberam a romifidina. Estes resultados estão de acordo com os resultados obtidos por MASSONE et al. (1988), nos quais observaram discreta bradipnéia, com espaços curtos (20 segundos) de pausa inspiratória, o que sugere depressão 


\begin{tabular}{|c|c|c|c|c|c|c|c|}
\hline \multirow[b]{2}{*}{ Parâmetro } & \multirow[b]{2}{*}{$\begin{array}{l}\text { Momentos } \\
\text { (minutos) }\end{array}$} & \multicolumn{2}{|c|}{ ROMIFIDINA } & \multicolumn{2}{|c|}{ ACEPROMAZINA } & \multicolumn{2}{|c|}{ DETOMIDINA } \\
\hline & & Média & DP & Média & DP & Média & DP \\
\hline \multirow{5}{*}{$\mathrm{pH}$} & 0 & 7,41 & 0,03 & 7,40 & 0,03 & 7,41 & 0,03 \\
\hline & 15 & 7,40 & 0,04 & 7,40 & 0,02 & 7,41 & 0,02 \\
\hline & 30 & 7,40 & 0,04 & 7,38 & 0,01 & 7,41 & 0,03 \\
\hline & 60 & 7,41 & 0,03 & 7,38 & 0,03 & 7,41 & 0,04 \\
\hline & 90 & 7,42 & 0,02 & 7,35 & 0,02 & 7,41 & 0,03 \\
\hline \multirow[t]{5}{*}{$\mathrm{PaO}_{2}$} & 0 & 96,90 & 7,28 & 113,95 & 9,85 & 85,07 & 15,80 \\
\hline & 15 & 90,80 & 7,31 & 111,87 & 33,47 & 86,02 & 21,59 \\
\hline & 30 & 95,90 & 9,07 & 89,25 & 12,16 & 88,15 & 11,07 \\
\hline & 60 & 93,30 & 8,89 & 98,30 & 17,94 & 86,86 & 15,40 \\
\hline & 90 & 96,00 & 9,31 & 103,75 & 0,64 & 77,58 & 11,39 \\
\hline \multirow[t]{5}{*}{$\mathrm{PaCO}_{2}$} & 0 & 36,78 & 1,84 & 35,57 & 2,55 & 40,66 & 2,00 \\
\hline & 15 & 38,06 & 3,18 & 36,37 & 1,96 & 40,70 & 4,42 \\
\hline & 30 & 37,97 & 1,97 & 41,02 & 2,68 & 41,55 & 2,71 \\
\hline & 60 & 39,65 & 1,63 & 41,37 & 3,11 & 43,01 & 3,15 \\
\hline & 90 & 38,69 & 1,84 & 40,65 & 5,30 & 42,35 & 2,65 \\
\hline
\end{tabular}

pH - no sangue arterial; $\mathrm{PaO}_{2}$ - pressão parcial de oxigênio no sangue arterial; $\mathrm{PaCO}_{2}$ pressão parcial de dióxido de carbono no sangue arterial;DP- desvio padrão.

arterial, cinco minutos após a administração da romifidina. MUIR \& HUBBELL (1991) observaram aumento da pressão parcial de dióxido de carbono no sangue arterial, decorrente da depressão respiratória, fato não constatado no presente estudo, pois nossa avaliação iniciou-se posteriormente. Apesar da obtenção de alguns valores superiores da $\mathrm{PaO}_{2}$, em alguns momentos de observação, não foram encontradas alterações clinicamente significativas em nenhum dos grupos estudados.

Não se observou excitação nos animais que receberam acepromazina, porém, a sedação observada com este agente não foi a desejada. Ao contrário, observou-se bons resultados com relação aos animais tratados com os $\alpha$ 2 agonistas, propiciando sedação adequada, o que vem ao encontro com os relatos de CLARKE et al.(1991) e

respiratória. No presente estudo, foi verificado diminuição da frequiência respiratória em relação aos valores basais, contudo, esta diminuição não foi estatistica ou biologicamente significativa.

Verificou-se no presente estudo, aumento significativo da pressão arterial, após 15 minutos da administração dos agentes $\alpha-2$ agonistas. Este mesmo efeito foi observado por WAGNER et al. (1991), atribuindo a hipertensão à estimulação alfa adrenérgica pós-sináptica periférica, resultando em vasoconstrição e aumento da pressão arterial. Efeito semelhante foi observado por MUIR \& MASON (1993) em relação a xilazina e a detomidina.

A acepromazina promoveu queda bastante significativa da pressão arterial em todos os tempos estudados, tanto na análise comparativa entre os grupos, como na análise comparativa dos diferentes tempos de observação de um mesmo grupo. Estes resultados vão ao encontro com os achados por MUIR \& SHEEHAN (1979). Porém, a taquicardia reflexa promovida pela diminuição da pressão arterial, observada por MUIR \& MASON (1993), não foi verificada neste experimento, mesmo com a queda acentuada da pressão arterial.

CLARKE $\boldsymbol{e t}$ al. (1991) verificaram diminuição da pressão parcial de oxigênio no sangue
ENGLAND $\boldsymbol{e t}$ al. (1992). Tal grupo farmacológico promove sedação mais confiável que os fenotiazínicos, além de serem analgésicos, o que não acorre com a acepromazina (MUIR e HUBBELL, 1991). A duração da sedação tem sido considerada uma das grandes vantagens quanto à utilização da romifidina. Em doses equipotentes, a sedação da romifidina tem duração mais longa do que a detomidina, o que vem ao encontro com os relatos de KANNEGIETER (1993).

Portanto, verificou-se no presente estudo que as alterações cardiovasculares promovidas pelos agentes $\alpha-2$ agonistas foram menos deletérias que aquelas desencadeadas pela acepromazina.

\section{FONTES DE AQUISIÇÃO}

${ }^{a}$ Acepran a 1\% - Univet Indústria Veterinária S.A.

${ }^{\mathrm{b}}$ Domosedan - Boheringer Ingelheim

${ }^{\mathrm{c}}$ Sedivet- Boheringer Ingelheim

${ }^{\mathrm{d}}$ Funbec TC 500- São Paulo

${ }^{\mathrm{e}}$ Imbracrios - São Paulo

${ }^{\mathrm{f}} \mathrm{BL} 330$ - Radiometer - Germany

\section{REFERÊNCIAS BIBLIOGRÁFICAS}

BOOTH, N.H., MCDONALD, L.E. Farmacologia e terapêutica em veterinária. 6.ed. Rio de Janeiro: Guanabara Koogan, 1992,cap. 16: Analgésicos não narcóticos. p. 262-288. 
CHAMBERS, J.P., LIVINGSTON, A., WATERMAN, A.E.,et al. Analgesic effects of detomidine in thoroughbred horses with chronic tendon injury. Research in Veterinary Science. v. 54, p. 52-56, 1993.

CLARKE, K.W., ENGLAND, G.C.W., GOOSSENS, L. Sedative and cardiovascular effects of romifidine, alone and in combination with butorphanol, in the horse. Journal of Veterinary Anaesthesia. v. 18, p. 25-30, 1991.

ENGLAND, G.C.W., CLARKE, K.W., GOOSSESNS, L.A comparision of the sedative effects of three alfa-2 adrenoceptor agonists (romifidine, detomidine and xylazine) in the horse. Journal Veterinary Pharmacology and Therapeutics, v. 15, p. 194-201, 1992.

FEITOSA, F.L.F., MARCONDES, M., MASSONE, F. Avaliação farmacológica da detomidina: um novo agente hipnoanalgésico para uso em eqüinos. Comunicações Científicas da Faculdade de Medicina Veterinária e Zootecnia da Universidade de São Paulo, v. 14, n. 2, p. 167-173, 1990.

GEISER, A.D. Chemical restraint and analgesia in the horse. Veterinary Clinics of North America: Equine Practice, v. 6. n. 3, p. 495-512, 1990

HAMM,D., JÖCHLE, W. Sedation and analgesia with Dormosedan (Detomidine Hydrochloride) or acepromazine for suturing of the vulvar lips in mares. Equine Veterinary Science, v. 11, n. 2, p. 86-88, 1991.
KANNEGIETER, N.J. The use of romifidine as a sedative in the horse. Australian Equine Veterinarian, v. 11, n. 2, p. 54-59, 1993.

LUMB, W.V., JONES, E.W. Veterinary anesthesia. 2.ed. Philadelphia: Lea \& Febiger, 1984, cap. 9, p. 165-195.

LUNA, S.P.L., VIEIRA, F.A.F., PAVANI, J. et al. Comparação entre detomidina e romifidina em eqüinos. A Hora Veterinária, n. 89, p. 56-60. 1990.

MASSONE, F., CASTRO, G.B., LUNA, S.P.L., et al. Estudo das diferentes doses de detomidina em eqüinos. A Hora Veterinária. n. 43, p. 13-20, 1988.

MUIR, W.W., HUBBELL, J.A.E. Equine Anesthesia. St Louis: Mosby-Year Book, 1991, cap. 11: Standing chemical restraint in horses: tranquilizers, sedatives, and analgesics, p. 247-280.

MUIR, W.W., MASON, D.E. Effects of diazepam, acepromazine, detomidine, and xylazine on thiamilal anesthesia in horses. Journal of the American Veterinary Medical Association, v. 203 , n. 7 , p. 1031-1038,1993.

MUIR, W.W., SHEEHAN, W. Hemodynamic and respiratort effects of a xylazine-acetylpromazine drug combination in horses. American Journal of Veterinary Research, v. 40, n. 10 , p. $1756-762,1979$

WAGNER, A.E., MUIR, W.W.; HINCHCLIFF,K.W. Cardiovascular effects of xylazine nd detomidine in horses. American Journal of Veterinary Research, v. 52, n. 5, p. 651-657, 1991.

Ciência Rural, v. 29, n. 1, 1999. 